\title{
PROCESS AND SYSTEMS Paying for what matters most: the future of outcomes-based payments in healthcare
}

\author{
Author: Rupert Dunbar-Rees ${ }^{\mathrm{A}}$
}

Payment by results' (PbR) is probably one of the most widely debated misnomers in the NHS. At best, rather than reimbursing results, it can be considered as payment for providing collections of acute care activities. Better prevention is often cited as the only solution to sustainability, but currencies which reward prevention seemingly remain very hard to create. This review article explores the impact of legacy payment systems on health and care systems nationally and internationally. It examines alternative models, such as payment for outcomes, and questions whether any alternative payment systems may better support prevention, and finally achieve the 'holy grail' of a truly sustainable care system. If payment for outcomes offers a potential solution, then what are the key barriers and enablers? The case for alternative, novel metrics for success is proposed, which may better support care systems to measure their progress towards sustainability than existing indicators.

KEYWORDS: Sustainability, healthcare outcomes, novel payment models, capitation, reimbursement

\section{Introduction \\ 'Payment by results' (PbR) is probably one of the most widely debated misnomers in the NHS. At best, rather than reimbursing results, it can be considered as payment for providing collections of acute care activities. That is not to say that the principal commissioning model in England relies on pure payment for activity, with an entirely 'fee for service' based payment mechanism, as still predominates in the USA. ${ }^{1}$ Commentators have questioned whether PbR, as currently applied in English NHS and care is fit for purpose, for current and future care needs. ${ }^{2}$ In fact, case-based payments (like PbR), pay for performance (eg Quality and Outcomes Framework [QOF], some Commissioning for Quality and Innovation [CQUIN] schemes), block payments and global capitation (eg primary care global sum) all exist in UK commissioning and reimbursement mechanisms. ${ }^{3}$ See Supplementary file 1 for a summary. Frequently, many of these currencies operate within a single care pathway (eg care for people with diabetes), or a single care setting (eg primary care).}

Author: ${ }^{\mathrm{A} C E O}$ Outcomes Based Healthcare, NHS Innovation Fellow, Outcomes Based Healthcare, London, UK
This somewhat baffling system of different healthcare payment currencies has largely simply evolved over time. It is remarkable that healthcare functions as effectively as it does, given the complexity of legacy payment and reimbursement systems. The important question remains as to what the impact of this is on the sustainability of health and care, and what alternative options exist, which may better align with best clinical practice, and what matters most to patients.

\section{Current commissioning models}

Current commissioning models using payment by activity (and payment for process) as the predominant reimbursement mechanism have been in operation in England without substantial change (despite periodic NHS structural reorganisation), since the purchaser-provider split was first introduced in the early 1990s. However, considering such a system from sustainability perspectives raises serious questions as to the long-term viability of current approaches to commissioning. When analysed using key dimensions of healthcare sustainability, ${ }^{4}$ which reflect the opposite of wasted resources from a number of perspectives, current commissioning models don't stand up well to scrutiny in terms of:

> Financial cost-effectiveness: with little underlying incentive for prevention, efficient care is not significantly rewarded under common payment by activity models. Indeed, the opposite is usually the case. For example, if providers are collectively successful in reducing the number of heart attacks and strokes in people with cardiovascular disease (CVD) for given area, the resulting loss in acute activity frequently penalises providers financially, in activity-based payment systems. Such approaches are fundamentally inconsistent with 'payment for prevention'.

$>$ Resource effectiveness:

$>$ infrastructure and fixed resources (eg hospitals, operating theatres etc), human resources (eg healthcare professionals): existing activity-based payment models do not adequately allow for effective use of these resources, since they encourage care to be predominantly delivered in the setting where the reimbursement is available/applies, even if cheaper, more efficient options exist ${ }^{5}$

> healthcare 'consumables' requiring large amounts of natural resources (eg drugs, devices etc): by focussing treatment predominantly on those already requiring care, rather than preventing the currently healthy population from developing illness, activity-based payment systems lead to unnecessary consumption of natural resource-intensive consumables ${ }^{3}$ 


\section{What we pay for now}

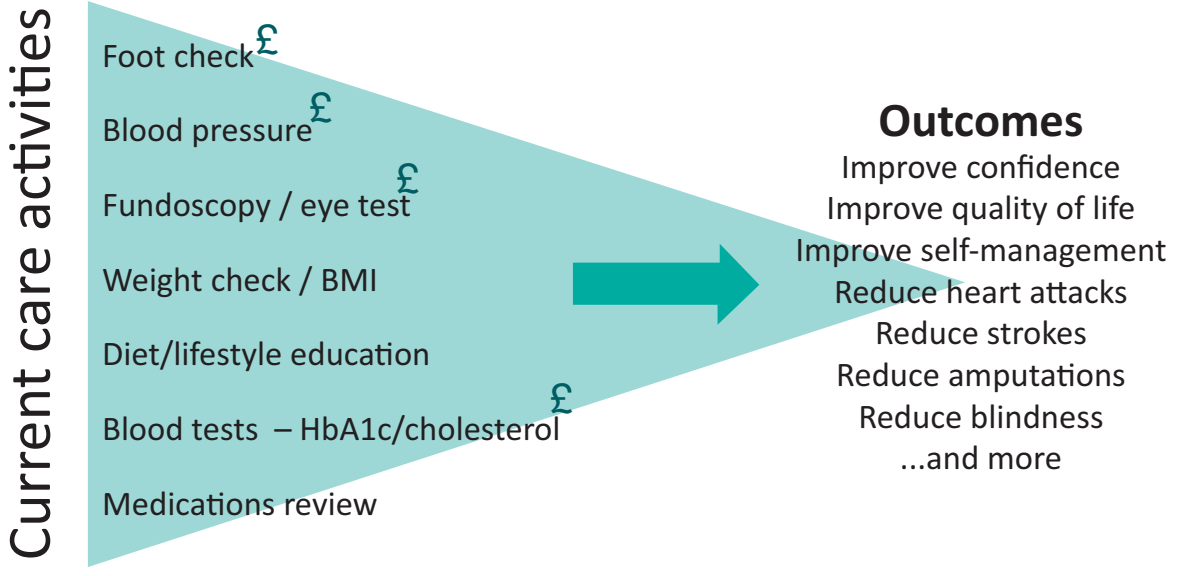

Fig 1. 'What we pay for now' Outcomes Based Healthcare slide. Adapted with permission from the following presentation at Digital Health Live, 5 May 2015, Dubai: Dunbar-Rees R, Hafezparast N. Reimbursement models that can enable the rapid advances happening in healthcare. Outcomes Based Healthcare 2015.

> environmental cost effectiveness: activity-based payment does not encourage care in less energy intensive settings closer to home or in the community. Given current policy imperatives, care closer to home may often actually be achieved in local areas, despite the commissioning/payment system, rather than because of some inherent characteristic of payment for activity. ${ }^{6}$

If commissioning models which rely on payment by activity don't appear to be sustainable, it is helpful to examine the underlying reasons, to avoid proposing changes which are equally unsustainable. The case for change from current commissioning models using activity or process-based payment can be summarised as follows (see the diabetes example in Fig 1 for illustration of the first, second and third points)

These approaches:

> 'lock in' money in existing care settings, even if alternative care settings are more appropriate, or more sustainable ${ }^{7}$

$>$ inhibit innovation; even if there is considerable evidence for a new innovation, unless the innovation is included in a payment mechanism tariff, the innovation will not be reimbursed, leading to slower, or even failed, adoption ${ }^{8}$

$>$ crowd out valuable care processes; not all evidence-based care processes and activities can be paid for using activity or processbased payment mechanisms - there are simply too many to keep track of. This leads to 'crowding out' of valuable care processes, or conditions requiring treatment, in favour of those that are reimbursed, as documented in the introduction of the QoF in the UK ${ }^{9}$

> fail to keep pace with guidelines and evidence; payment mechanisms take a long time to 'catch up' with best clinical practice. By definition, best clinical practice for any condition or pathway should be updated on a daily, or weekly, basis. It is unrealistic to expect any activity-based payment system to be able to evolve at the same pace as changes to the evidencebase, in all conditions and pathways. There is considerable evidence that by the time new evidence has been incorporated into payment mechanisms, the evidence is already out of date $^{10}$

$>$ frequently fail to eliminate low value care; activity-based payment does not encourage the elimination of low value (potentially harmful) interventions, resulting in allocative inefficiency and even avoidable harm ${ }^{11}$

> focus predominantly (or exclusively) on those already needing treatment; largely focuses on care provision and resource allocation for those already with long-term / complex conditions, rather than maintaining the currently healthy population through effectively targeted prevention activities (even if the evidence demonstrates that money is better spent there)

> are failing to adequately address health inequalities; with variations in life expectancy, healthy life expectancy and health outcomes indicators representing a range of health inequalities are still widening over recent years, corresponding to deprivation levels. ${ }^{12,13}$

\section{Alternative commissioning and/or payment models which underpin novel care models}

Before exploring whether alternative commissioning models underpinned by novel payment mechanisms could support more sustainable care, it is first necessary to reflect on the sometimes confusing and inconsistently applied terminology often associated with alternative payment models. Despite considerable progress from leading commentators in defining value, there remains confusion as to what 'value' in healthcare means, from the perspectives of different stakeholders, and between different countries. ${ }^{9,14,15}$ The terms 'value-based payment' and 'outcomesbased payment' are often used interchangeably and generally mean payment to providers for achieving better outcomes. This sometimes extends to payment for the activities and care processes which support improvements in those outcomes. Occasionally current activity-based payment approaches are rebadged as 'value-based payments' or 'outcome-based payments' 
at a local level, while inputs or care processes are renamed as 'outcomes'. This doesn't help the case for novel or alternative payment models and puts adoption of these (alternative models) at risk.

It is worth noting that outcomes-based payments cannot exist in isolation. It is not practical or desirable for all activity-based payment to be switched to outcomes-based payments; not all adverse outcomes are avoidable and not all good outcomes are achievable. Furthermore, confounding effects and levels of patient activation all require careful consideration. ${ }^{16}$ Complex technical challenges perhaps, but that is not to say that these considerations are any less resolvable than the current complexity of activity-based payments systems. However, all outcomes-based payments generally need to operate alongside some element of payment which accounts for the number of people receiving care.

Outcomes-based payments can operate alongside activitybased payment systems; however, care needs to be taken to manage any potential conflicts between the two. More typically, health systems are seeking to implement capitated payments, or closely related 'bundled payments' (which operate at individual condition level), alongside outcomes-based payments. ${ }^{3,17}$ Capitated payment, or simply 'capitation', refers to payment to a provider or group of providers, to cover the majority (or all) of the care provided to a specified population, for a specified period of time, across different care settings. ${ }^{3,18}$ This is then subject to contingent payments, depending on whether outcomes have been improved or not, with some commentators suggesting that incentives between $30 \%$ and $50 \%$ of the total are needed to promote change. ${ }^{19}$ In the NHS in England, and underpinning the recently-branded Integrated Care Systems, ${ }^{20}$ the capitated element is being called a 'Whole population budget' (WPB), while the outcomes-based payment element is referred to as an 'Improvement payment scheme' (IPS). ${ }^{21}$ Critically important to understanding the operation of the model, both the WPB and IPS operate at an 'Integrated care system' level, ie spanning multiple providers, rather than being specific to individual providers or care settings.

Critics of such approaches may argue that payment for outcomes is simply another form of 'Pay for performance'
(P4P); a policy with mixed evidence when implemented in practice. ${ }^{3,22}$ However, what matters in P4P schemes is what kind of performance is being incentivised. Incentivisation of care processes and specific activities can result in 'tick-box' or even 'one-size-fits-all' medicine, which is in no-one's best interests and is subject to the problems identified above..$^{23,24}$

\section{The case for outcomes as a 'health currency'}

As metrics of care quality, outcomes tend to be much more stable over time than quality process measures; instead of requiring updating for each new piece of evidence, what matters most to patients remains remarkably stable. For example, as well as personal and functional outcomes (like quality of life and confidence), avoiding adverse outcomes such as myocardial infarction (MI), stroke, amputation, kidney failure or blindness will remain important outcomes for people with diabetes for some time. That is irrespective of the ever-changing, precise combinations of care processes, treatments and lifestyle changes necessary to avoid such complications.

Furthermore, at an individual patient level, healthcare professionals may feel a greater level of autonomy (within recognised, evidence-based guidelines) to use whatever combination of care processes are most appropriate for the patient's individual circumstances, without worrying about whether they are the reimbursed care processes, or not. What is prioritised is whether the outcome was improved, rather than commissioners attempting to mandate providers on the precise 'how', for each individual patient. This also incentivises providers and healthcare professionals to innovate (assuming there is an evidence base, or rationale); better outcomes will always be rewarded, irrespective of whether there is a tariff for the treatment or not (see Fig 2 for illustration of the impact on innovation of new, outcomes-based, health currencies).

Both capitation and outcomes-based payments may support the evolution of new, more sustainable, care models through intrinsic 'payment for prevention'. When measured at a population level, providers in integrated care systems could be paid when the population with CVD avoids or reduces heart attacks, rather
Fig 2. 'What we need - new health currencies' Outcomes Based Healthcare slide. Adapted with permission from the following presentation at Digital Health Live, 5 May 2015, Dubai: DunbarRees R, Hafezparast N. Reimbursement models that can enable the rapid advances happening in healthcare. Outcomes Based Healthcare, 2015.

\section{What we need: new health currencies}

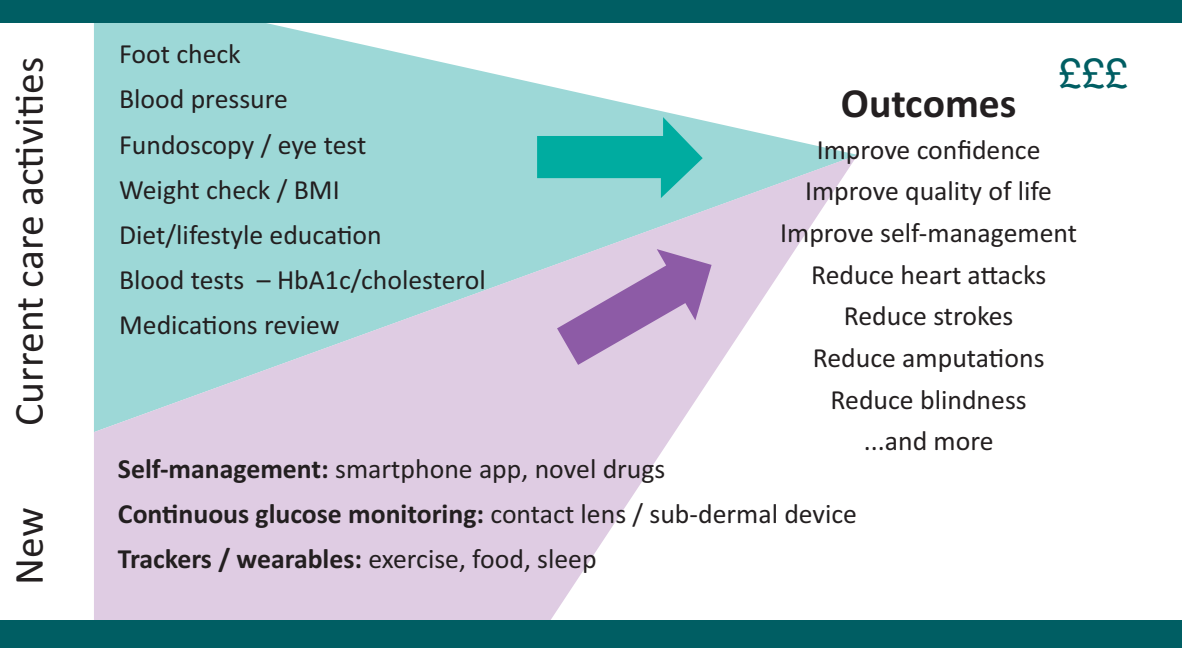


than only when individual people are treated for heart attacks, as an example. Separately, under a capitation and outcomes-based model, if care is more efficiently delivered closer to home, requiring fewer human and natural resources, then the benefits are not diluted by mandated use of activity-based payment designed for acute settings. Of course, this necessitates accurate, timely analysis and monitoring at a population level, as well as careful understanding of historical baselines, trajectories, with accurate measures of demographic change over time - none of which are insurmountable.

\section{Barriers and enablers to adoption}

For outcomes-based payments to truly take off, there are some challenges around creation of suitable datasets, accurate measurement, standardisation, case-mix adjustment, and attribution to navigate, but many areas in the UK and internationally are well advanced in implementing these models. This demonstrates that it is not only possible, but it is actually happening. ${ }^{25,26}$

A focus on value and outcomes is essential, but also needs to encompass allocative value, rather than focusing discreetly on those with defined medical conditions, in the way existing approaches can do. To fully establish value, social, societal and environmental costs of care need to be considered. By definition these need to include assessment of costs and impacts on all the population as a whole, and not just the person currently receiving treatment. Without these, there will only ever be a partial assessment of value in healthcare. Underpinning all of this, and in order to organise care effectively, effective approaches to population segmentation are required, recognising groups of people with similar health and care needs, around which to organise services. ${ }^{27-29}$

Ultimately, even outcomes measures need to evolve to measure more sustainable care. Novel population metrics are required, which reflect the proportion of a person's life span which is spent predominantly healthy (ie improving a person's 'healthspan'), rather than focusing exclusively on lifespan. Healthcare is reasonably well served for metrics of secondary prevention, but by then the best opportunity has been lost. Measures such as 'healthspan', would make excellent markers of the success of primary prevention, and are essential to the achieving sustainability of health and care systems.

\section{Conclusion}

It would be unhelpful, and misses the point entirely, if alternative commissioning models were reduced to simply a technical exercise about financial allocations or reimbursement mechanisms. Underneath all of the unhelpful language and financial complexity, healthcare should strive for systems of care which are organised and integrated around groups of patients (families, carers, service users) with similar needs, rather than being organised largely around provider requirements or organisational characteristics. Among other things, that means routinely recognising, accounting for, and proactively managing comorbidity, rather than 'risk adjusting' for it, or referring onward to care from different specialties for each condition separately. The opportunity is significant, while the risks are serious if we choose not to put health and care on a more sustainable footing. Commissioning and payment mechanisms that underpin moves to sustainable care are essential enablers to get right. Health and care systems focused on simply delaying death are no longer sustainable. Unless improvements in life expectancy are more than matched by improvements in people's 'healthspans', then lifetime costs of care will only continue to escalate unsustainably. However, it is extremely hard to improve what is not measured. In this context, the need to develop and agree on a definitive, population-level measure of 'healthspan' has never been more pressing.

\section{Supplementary material}

Additional supplementary material may be found in the online version of this article at http://futurehospital.rcpjournal.org:

S1 - Reimbursement models - key characteristics

\section{References}

1 Rama A. Policy Research Perspectives. Payment and Delivery in 2016: The Prevalence of Medical Homes, Accountable Care Organizations, and Payment Methods Reported by Physicians. American Medical Association, 2017.

2 Appleby ], Harrison T, Hawkins L, Dixon A. Payment by Results. How can payment systems help to deliver better care? The Kings Fund, 2012.

3 Bersani J, Dunbar-Rees R, McGough R. Contracting for outcomes. Outcomes Based Healthcare. London: Outcomes Based Healthcare, 2014.

4 Hazell B, Robson R. Pharmaceutical waste reduction in the NHS. NHS England, 2015.

5 The Newcastle upon Tyne Hospitals NHS Foundation Trust. Sustainable Healthcare Strategy 2016-2020. [Accessed 19 March 2018].

6 Albury D, Beresford T, Dew S et al. Against the odds: Successfully scaling innovation in the NHS. Innovation Unit and The Health Foundation, 2018.

7 Gillam SJ, Siriwardena AN, Steel N. Pay-for-performance in the United Kingdom: impact of the quality and outcomes framework: a systematic review. Ann Fam Med 2012;10:461-8.

8 Bloor C, Sriskandarajah R, Croxford V, White H. Strategy for UK Life Sciences. Department for Business Innovation and Skills, Office for Life Sciences, 2011.

9 Gray M. How to get better value healthcare, 2nd edn. Oxford Press, 2011.

10 Department of Health. Annual report and accounts 2016 to 17. London: DH, 2017.

11 Buck D. Reducing inequalities in health: towards a brave old world? The Kings Fund, 2017. www.kingsfund.org.uk/blog/2017/08/ reducing-inequalities-health-towards-brave-old-world [Accessed 19 March 2018].

12 Cooper Z, Gibbons S, Jones S, McGuire A. Does hospital competition save lives? Evidence from the English NHS Patient Choice Reforms. The Economic Journal 2011:554;228-60.

13 Holmstrom B, Milgrom P. Multitask principal-agent analyses: incentive contracts, asset ownership and job design. Journal of Law, Economics and Organization 1991;7:24-52.

14 Porter M. What is value in health care? NEJM 2010;363:2477-81.

15 Pendleton RC. We won't get value-based health care until we agree on what 'value' means. Harvard Business Review, 2018. https://hbr. org/2018/02/we-wont-get-value-based-health-care-until-we-agreeon-what-value-means [Accessed 19 March 2018].

16 Hibbard J, Gilburt H. Supporting people to manage their health-an introduction to patient activation. The Kings Fund, 2014.

17 Porter ME, Kaplan RS. How to pay for health care. Harvard Business Review 2016. 
18 Monitor, NHS England. Capitation: an introduction. Monitor, NHS England, 2015.

19 Latkovic T. The trillion dollar prize. Using outcomes-based payment to address the US healthcare financing crisis. McKinsey \& Company, 2013.

20 NHS England. Whole population models of provision: Establishing integrated budgets. NHS England, 2017.

21 NHS England. Finance and Payment Approach for ACOs. NHS England, 2017.

22 Cashin C, Chi Y, Smith P, Borowitz M, Thomson S. Paying for Performance in Health Care. Implications for health system performance and accountability. European Observatory on Health Systems and Policies, 2014.

23 Tipirneni R, McMahon LF. Measuring value in primary care: enhancing quality or checking the box? Health Serv Res 2014:49:1724-8

24 Lester H, Matharu T, Mohammed MA, Lester D, Foskett-Tharby R. Implementation of pay for performance in primary care: a qualitative study 8 years after introduction. Br J Gen Pract 2013;63:e408e415.

25 Healthcare Financial Management Association. New care models programme and vanguard sites: summary for members. HFMA, 2016.
26 Integrated Care Systems. NHS England. www.england.nhs.uk/ accountable-care-systems/ [Accessed 19 March 2018].

27 Lynn J, Straube BM, Bell KM, Jencks SF, Kambic RT. Using population segmentation to provide better health care for all: the 'Bridges to Health' model. The Milbank Quarterly 2007;85:185-208.

28 Outcomes Based Healthcare. Whole Population Segmentation Models for Outcomes Measurement. OBH, 2016. https:// outcomesbasedhealthcare.com/evaluation-of-whole-populationsegmentation-models/

29 Outcomes Based Healthcare. Evaluation of the Bridges to Health Segmentation Model for Outcomes Measurement. OBH, 2016.

Address for correspondence: Dr Rupert Dunbar-Rees, Outcomes Based Healthcare, 11-13 Cavendish Square, London W1G OAN, UK.

Email: rupsdr@outcomesbasedhealthcare.com 